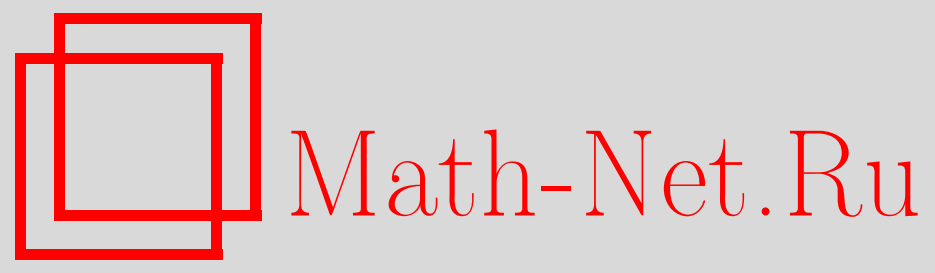

Д. А. Тимушев, О скорости сходимости по вероятности спектральной функции распределения случайной матрицы, Теория вероятн. и ее примен., 2006, том 51, выпуск 3, 618-622

DOI: https://doi.org/10.4213/tvp45

Использование Общероссийского математического портала Math-Net.Ru подразумевает, что вы прочитали и согласны с пользовательским соглашением

http: //www . mathnet.ru/rus/agreement

Параметры загрузки:

IP : 54.157 .27 .8

26 апреля 2023 г., $15: 49: 24$

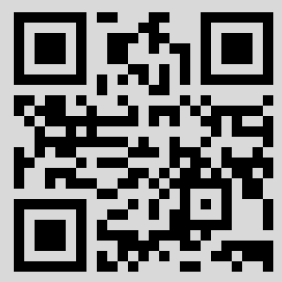


10. Monat P., Stricker C. Fölmer-Schweizer decomposition and mean-variance hedging for general claims. - Ann. Probab., 1995, v. 23, № 2, p. 605-628.

11. Föllmer H., Schweizer $M$. Hedging of contingent claims under incomplete information. - Applied Stochastic Analysis (London, 1989). Ed. by M.H. A. Davis and R. J. Elliott. Stochastics Monogr., v. 5. New York: Gordon and Breach, 1991, p. 389414.

12. Ширяев $A$. Н. Основы стохастической финансовой математики. Т. 2: Теория. М.: ФАЗИС, 1998, 544 c.

13. Musiela M., Rutkowski M. Martingale Methods in Financial Modelling. Berlin: Springer-Verlag, 1997, $512 \mathrm{p}$.

14. Эллиотm P. Стохастический анализ и его приложения. М.: Мир, 1986, 352 c.

15. Schäl M. On quadratic cost criteria for option hedging. - Math. Oper. Res., 1994, v. 19, № 1, p. 121-131.

16. Schweizer $M$. Variance-optimal hedging in discrete time. - Math. Oper. Res., 1995, v. 20, № 1, p. 1-32.

Поступила в редакцию

19.VIII.2004

(C) 2006 r.

ТИМУШЕВ Д. А.*

\section{О СКОРОСТИ СХОДИМОСТИ ПО ВЕРОЯТНОСТИ СПЕКТРАЛЬНОЙ ФУНКЦИИ РАСПРЕДЕЛЕНИЯ СЛУЧАЙНОЙ МАТРИЦЫ ${ }^{1)}$}

Показано, что расстояние Колмогорова между спектральной функцией распределения $(n \times n)$-матрицы из GUE и функцией распределения полукругового закона имеет стохастический порядок $O_{p}\left((\ln n) / n^{2 / 3}\right)$.

Ключевые слова и фразы: случайная матрица, полукруговой закон, вигнеровский ансамбль, гауссовский унитарный ансамбль.

Рассмотрим вигнеровскую эрмитову случайную матрицу $\mathbf{W}=\left(w_{k j}\right)$ размерности $n \times n$. По определению ансамбля Вигнера, элементы $w_{k j}, 1 \leqslant k \leqslant j \leqslant n$, являются независимыми одинаково распределенными комплексными случайными величинами, с независимыми мнимыми и вещественными частями, причем $\mathbf{E} w_{k j}=0$ и $\mathbf{E}\left|w_{k j}\right|^{2}=\sigma^{2}$. Обозначим $\lambda_{j}, 1 \leqslant j \leqslant n$, собственные числа матрицы $n^{-1 / 2} \mathbf{W}$, а $F_{n}(x)=n^{-1} \#\left\{j: \lambda_{j} \leqslant x\right\}$ спектральную функцию распределения матрицы $n^{-1 / 2} \mathbf{W}$. Пусть $G(x)$ - функция распределения полукругового закона с плотностью

$$
G^{\prime}(x)=\frac{1}{2 \pi \sigma^{2}} \sqrt{4 \sigma^{2}-x^{2}} \mathbf{I}_{\{|x| \leqslant 2 \sigma\}} .
$$

Нас будет интересовать скорость сходимости спектральной функции $F_{n}(x)$ к функции распределения $G(x)$ в метрике Колмогорова, а также в метриках пространств $L_{1}$ и $L_{2}$, т.е. величины

$$
\begin{gathered}
\Delta_{n}^{*}:=\sup _{x}\left|F_{n}(x)-G(x)\right| \\
L_{p}\left(F_{n}, G\right):=\left(\int_{-\infty}^{\infty}\left|F_{n}(x)-G(x)\right|^{p} d x\right)^{1 / p}, \quad p=1,2 .
\end{gathered}
$$

* Математический факультет, Сыктывкарский государственный университет, Октябрьский пр., 55, 167001 Сыктывкар, Россия.

1) Работа выполнена при поддержке DFG-Forshergruppe FOR 399/1 и SFB 701 Universität Bielefeld. 
Скорость сходимости к полукруговому закону оценивалась в работах многих авторов (см., например, [2]-[5] и литературу к ним). В [3], в частности, показано, что для вигнеровских матриц, четвертые моменты элементов которых равномерно ограничены, имеет место оценка

$$
\Delta_{n}:=\sup _{x}\left|\mathbf{E} F_{n}(x)-G(x)\right|=O\left(n^{-1 / 2}\right)
$$

для скорости сходимости ожидаемой спектральной функции $\mathbf{E} F_{n}(x)$ к функции распределения $G(x)$. А при равномерной ограниченности двенадцатых моментов, аналогичная оценка $\mathbf{E} \Delta_{n}^{*}=O\left(n^{-1 / 2}\right)$ показана для сходимости по вероятности спектральной функции $F_{n}(x)$ к функции распределения $G(x)$. В данной работе мы рассматриваем более узкий, чем вигнеровский, класс матриц - гауссовский унитарный ансамбль (GUE). Это вигнеровские матрицы, элементы которых $w_{k j}$ имеют гауссовское распределение. В этом случае известно точное аналитическое представление совместной плотности распределения собственных чисел и, как следствие, плотности ожидаемой спектральной функции $\left(\mathbf{E} F_{n}(x)\right)^{\prime}$ через функции Эрмита, что позволяет воспользоваться при оценивании скорости сходимости соответствующей асимптотикой. Сходимость ожидаемой спектральной функции матриц из GUE исследовалась в работе [5], где была получена оптимальная оценка $\Delta_{n}=O\left(n^{-1}\right)$. В то же время, для сходимости по вероятности известен лишь общий результат, упомянутый выше. В этой работе нами получен более точный результат.

Для удобства положим $\sigma^{2}=\frac{1}{4}$. Символ $C$, с индексом или без него, на протяжении всей работы будет обозначать абсолютные положительные константы, необязательно одни и те же в разных формулах. Имеет место следующая теорема.

Теорема 1. Существует такая положительная константа $C$, ито

$$
\begin{aligned}
\mathbf{E} L_{p}\left(F_{n}, G\right) & \leqslant C \frac{\sqrt{\ln n}}{n}, \quad p=1,2, \\
\mathbf{E} \Delta_{n}^{*} & \leqslant C \frac{\ln n}{n^{2 / 3}} .
\end{aligned}
$$

Д о к а з а т е л ь с т в о. Мыначнем с доказательства соотношения (1). Положим $\varepsilon_{n}=C n^{-2 / 3}$. Из показанного в работе [5] неравенства

$$
\sup _{-1+\varepsilon_{n} \leqslant x \leqslant 1-\varepsilon_{n}}\left|\mathbf{E} F_{n}(x)-G(x)\right| \leqslant C n^{-1}
$$

и из конечности носителя плотности полукругового закона $G^{\prime}(x)$ следует, что

$$
L_{p}\left(\mathbf{E} F_{n}, G\right) \leqslant \frac{C}{n}, \quad p=1,2 .
$$

Поэтому нам достаточно оценить величины

$$
\mathbf{E} L_{p}\left(F_{n}, \mathbf{E} F_{n}\right), \quad p=1,2 .
$$

Рассмотрим сначала случай $p=2$.

Пусть

$$
K_{n}(s, t)=\left(\frac{n}{2}\right)^{1 / 2} \frac{\varphi_{n}(s) \varphi_{n-1}(t)-\varphi_{n-1}(s) \varphi_{n}(t)}{s-t}
$$

обозначает ядро Кристоффеля-Дарбу,

$$
p_{n}(s)=\left(\frac{2}{n}\right)^{1 / 2} K_{n}(\sqrt{2 n} s, \sqrt{2 n} s)
$$

- плотность ожидаемой спектральной функции распределения $\mathbf{E} F_{n}(x)$ (cм. [6]). Здесь $\left\{\varphi_{i}\right\}$ обозначают функции Эрмита. Пусть $x \in \mathbf{R}$. Несложно проверить, что

$$
\mathbf{E}\left(F_{n}(x)-\mathbf{E} F_{n}(x)\right)^{2}=\frac{2}{n} \int_{-\infty}^{x} \int_{x}^{\infty} K_{n}^{2}(\sqrt{2 n} s, \sqrt{2 n} t) d s d t .
$$


Таким образом, нам достаточно показать, что

$$
\int_{-\infty}^{\infty} \int_{-\infty}^{x} \int_{x}^{\infty} K_{n}^{2}(\sqrt{2 n} s, \sqrt{2 n} t) d t d s d x=O\left(\frac{\ln n}{n}\right) .
$$

Для этого воспользуемся асимптотикой Планшереля-Ротача для функций Эрмита (см., например, $[1$, с. 700]), которая может быть представлена в виде следующей ниже таблицы:

\begin{tabular}{|c|c|}
\hline$s$ & $\varphi_{n}(\sqrt{2 n} s)$ \\
\hline$-1+\varepsilon_{n} \leqslant s \leqslant 1-\varepsilon_{n}$ & $O\left(n^{-1 / 4}\left(1-s^{2}\right)^{-1 / 4}\right)$ \\
\hline $1-\varepsilon_{n} \leqslant s \leqslant 1+\varepsilon_{n}$ & $O\left(n^{-1 / 12}\right)$ \\
\hline $1+\varepsilon_{n} \leqslant s \leqslant \sqrt{2}$ & $O\left(n^{-1 / 4}(s-1)^{-1 / 4} e^{-C n(s-1)^{3 / 2}}\right)$ \\
\hline$s \geqslant \sqrt{2}$ & $O\left(e^{-C n s^{2}}\right)$ \\
\hline
\end{tabular}

Пользуясь данной асимптотикой и неравенством (3), получим требуемое.

Перейдем к доказательству соотношения (1) при $p=1$. Легко убедиться, что

$$
\begin{aligned}
\mathbf{E} L_{1}\left(F_{n}, \mathbf{E} F_{n}\right) \leqslant & 2 \mathbf{E} L_{2}\left(F_{n}, \mathbf{E} F_{n}\right)+2 \int_{\sqrt{2}}^{\infty}\left(1-\mathbf{E} F_{n}(x)\right) d x \\
& +2 \int_{-\infty}^{-\sqrt{2}} \mathbf{E} F_{n}(x) d x \leqslant 4 \int_{-\infty}^{-\sqrt{2}} \int_{-\infty}^{x} p_{n}(s) d s d x+C \frac{\sqrt{\ln n}}{n} .
\end{aligned}
$$

Последнее неравенство является следствием (1) при $p=2$. Из асимптотики функций Эрмита заключаем, что

$$
\int_{-\infty}^{-\sqrt{2}} \int_{-\infty}^{x} p_{n}(s) d s d x \leqslant C \sqrt{n} \int_{-\infty}^{-\sqrt{2}} \int_{-\infty}^{x} e^{-C n s^{2}} d s d x \leqslant \frac{C}{n \sqrt{n}} e^{-C n} .
$$

Тем самым доказательство соотношения (1) завершено.

Покажем теперь справедливость (2).

Обозначим $t_{n}(z)$ и $s(z)$ преобразования Стилтьеса спектральной функции распределения $F_{n}(x)$ и функции распределения полукругового закона $G(x)$ соответственно. Тогда

$$
s(z)=\int_{-\infty}^{\infty} \frac{1}{x-z} d G(x)=-2 z+2 \sqrt{z^{2}-1}, \quad t_{n}(z)=\int_{-\infty}^{\infty} \frac{1}{x-z} d F_{n}(x) .
$$

Введем обозначения:

$$
\delta_{s} t_{n}(u, v):=t_{n}(u+i v)-s(u+i v), \quad \delta_{E} t_{n}(u, v):=t_{n}(u+i v)-\mathbf{E} t_{n}(u+i v) .
$$

Пусть $v$ и $a$ - положительные числа, $x^{\prime}=x+v a$. Следуя работе [3], можно показать, что найдутся такие положительные константы $C_{1}, C_{2}, C_{3}, C_{4}, C_{5}(a)$, что для всех $V>v$ и всех $x \in \mathbf{R}$

$$
\left|F_{n}(x)-G(x)\right| \leqslant C_{1} A_{1}+C_{2} A_{2}\left(x^{\prime}\right)+C_{3} A_{3}+C_{4} \Delta_{n}+C_{5}(a) v,
$$

где

$$
A_{1}=\int_{-\infty}^{\infty}\left|\delta_{s} t_{n}(u, V)\right| d u, \quad A_{2}\left(x^{\prime}\right)=\left|\int_{v}^{V} \delta_{s} t_{n}\left(x^{\prime}, u\right) d u\right|, \quad A_{3}=\frac{1}{\sqrt{v}} L_{2}\left(F_{n}, \mathbf{E} F_{n}\right)
$$

Введем в рассмотрение величины

$$
x_{k}=-1+\frac{2 k}{n}, \quad x_{k}^{\prime}=x_{k}+v a, \quad 0 \leqslant k \leqslant n .
$$


Тогда

$$
\mathbf{E} \Delta_{n}^{*} \leqslant C_{1} \mathbf{E} A_{1}+C_{2} \mathbf{E} \max _{0 \leqslant k \leqslant n} A_{2}\left(x_{k}^{\prime}\right)+C_{3} \mathbf{E} A_{3}+C_{4} \Delta_{n}+C_{5}(a) v+C n^{-1} .
$$

Далее будем полагать, что $V=1$ и $v=C n^{-2 / 3}$.

Легко заметить, что соотношение (1) при $p=2$ влечет оценку $\mathbf{E} A_{3}=$ $O\left((\ln n) / n^{2 / 3}\right)$. Из работы $[3,(4.30)]$ следует неравенство

$$
\int_{-\infty}^{\infty}\left|\mathrm{E} \delta_{s} t_{n}(u, V)\right| d u \leqslant \frac{C}{n},
$$

которое, вместе с соотношением (1) при $p=1$ дает $\mathbf{E} A_{1}=O((\ln n) / n)$. Таким образом, для завершения доказательства (2) нам остается доказать оценку

$$
\mathrm{E} \max _{0 \leqslant k \leqslant n} A_{2}\left(x_{k}^{\prime}\right)=O\left(\frac{\ln n}{n^{2 / 3}}\right) .
$$

Из следствия 2.4 работы [7] имеем неравенство

$$
\mathbf{E}\left|\int_{v}^{V} \delta_{E} t_{n}\left(x_{k}^{\prime}, u\right) d u\right|^{2 p} \leqslant(C p)^{p} \mathbf{E}\left|\nabla \int_{v}^{V} t_{n}\left(x_{k}^{\prime}+i u\right) d u\right|^{2 p} .
$$

Принимая во внимание этот факт, можно показать, что найдется такая положительная константа $C$, что для всех $p \geqslant 1$ и $0 \leqslant k \leqslant n$ имеет место неравенство

$$
\mathbf{E}\left|\int_{v}^{V} \delta_{E} t_{n}\left(x_{k}^{\prime}, u\right) d u\right|^{2 p} \leqslant \frac{(C p)^{p}}{n^{2 p} v^{p}}
$$

Введем события

$$
\Omega_{k}=\left\{\left|\int_{v}^{V} \delta_{E} t_{n}\left(x_{k}^{\prime}, u\right) d u\right| \leqslant \frac{\ln n}{n \sqrt{v}}\right\} .
$$

Несложно убедиться, что для любых $0 \leqslant k \leqslant n, q \geqslant 1$ и достаточно больших $n$ выполнено неравенство $\mathbf{P}\left\{\bar{\Omega}_{k}\right\} \leqslant n^{-q}$. Учитывая это, имеем

$$
\begin{aligned}
\mathbf{E} \max _{0 \leqslant k \leqslant n} A_{2}\left(x_{k}^{\prime}\right) & \leqslant \mathbf{E} \max _{0 \leqslant k \leqslant n}\left|\int_{v}^{V} \delta_{E} t_{n}\left(x_{k}^{\prime}, u\right) d u\right|+C \frac{\ln n}{n} \\
& \leqslant \frac{\ln n}{n \sqrt{v}}+C \ln n \sum_{k=0}^{n} \mathbf{P}\left\{\bar{\Omega}_{k}\right\}+C \frac{\ln n}{n} \leqslant \frac{\ln n}{n^{2 / 3}} .
\end{aligned}
$$

Теорема доказана.

Автор выражает признательность проф. А.Н. Тихомирову и проф. Ф. Гётце за постановку задачи и полезные дискуссии.

\section{СПИСОК ЛИТЕРАТУРЫ}

1. Askey R., Wainger S. Mean convergence of expansions in Laguerre and Hermite series. - Amer. J. Math., 1965, v. 87, p. 695-708.

2. Bai $Z$. D. Methodologies in spectral analysis of large-dimensional random matrices, a review. - Statist. Sinica, 1999, v. 9, № 3, p. 611-677.

3. Götze F., Tikhomirov A. Rate of convergence to the semi-circular law. - Probab. Theory Related Fields, 2003, v. 127, № 2, p. 228-276.

4. Götze F., Tikhomirov A. Rate of convergence to the semi-circular law for the Gaussian unitary ensemble. - Теория вероятн. и ее примен., 2002, т. 47, в. 2, с. 381-387.

5. Götze F., Tikhomirov A. The rate of convergence for spectra of GUE and LUE matrix ensemble. - Cent. Eur. J. Math., 2005, v. 3, № 4, p. 666-704 (electronic).

6. Mehta M. L. Random Matrices, 3ed. Pure and Applied Mathematics (Amsterdam), v. 142. Amsterdam: Elsevier/Academic Press, 2004, 708 p. 
7. Pisier G. Probabilistic methods in the geometry of Banach spaces, Probability and analysis (Varenna, 1985). Lecture Notes in Math., v. 1206. Berlin: Springer-Verlag, 1986, p. 167-241.

Поступила в редакцию

13.VI.2006

(c) 2006 r.

ШЕВЦОВА И. Г.*

\section{УТОЧНЕНИЕ ВЕРХНЕЙ ОЦЕНКИ АБСОЛЮТНОЙ ПОСТОЯННОЙ В НЕРАВЕНСТВЕ БЕРРИ-ЭССЕЕНА ${ }^{1)}$}

Для абсолютной константы в классическом неравенстве БерриЭссеена для сумм независимых одинаково распределенных случайных величин, имеющих конечные моменты третьего порядка, получена оценка $C \leqslant 0,7056$.

Ключевые слова и фразы: центральная предельная теорема, нормальная аппроксимация, неравенство Берри-Эссеена, оценка скорости сходимости.

1. Постановка задачи и ее история. Пусть $X_{1}, \ldots, X_{n}$ - независимые одинаково распределенные случайные величины, удовлетворяющие условиям

$$
\begin{gathered}
\mathbf{E} X_{1}=0, \quad \mathbf{D} X_{1}=1 . \\
\beta^{3} \equiv \mathbf{E}\left|X_{1}\right|^{3}<\infty .
\end{gathered}
$$

Тогда, согласно неравенству Берри-Эссеена, для равномерного расстояния $\rho$ между функцией распределения $F_{n}(x)$ нормированной суммы $\left(X_{1}+\cdots+X_{n}\right) / \sqrt{n}$ и стандартной нормальной функцией распределения $\Phi(x)$ справедлива оценка

$$
\rho \equiv \sup _{x}\left|F_{n}(x)-\Phi(x)\right| \leqslant C \cdot \varepsilon
$$

где

$$
\varepsilon=\frac{\beta^{3}}{\sqrt{n}}
$$

а $C$ - положительная абсолютная постоянная [9], [10]. История отыскания значения абсолютной константы в неравенстве Берри-Эссеена интересна и богата результатами. Так, Э. Берри [9] утверждал, что $C \leqslant 1,88$, однако, как обнаружил позднее П. Л. Сюй [12], вычисления Берри содержали ошибку. К.-Г. Эссеен [10] показал, что $C \leqslant 7,59$. Х. Бергстрем [8] показал, что $C \leqslant 4,8$. К. Такано [14] получил оценку $C \leqslant 2,031$. По-видимому, работа Такано (опубликованная на японском языке) выпала из поля зрения некоторых исследователей, так как в нескольких более поздних публикациях приводятся немного худшие оценки. В частности, в работе [11] имеется упоминание о неопубликованных вычислениях, даюших $C \leqslant 2,9$. В работе Д. Л. Уоллеса [15] приведена оценка $C \leqslant 2,05$. В. Феллер [4], упоминая результат Уоллеса, также обходит вниманием работу Такано. Вычислению наименьшего возможного значения абсолютной постоянной $C$ придавал большое значение А. Н. Колмогоров.

* Московский государственный университет им. М.В. Ломоносова, факультет вычислительной математики и кибернетики, Ленинские горы, 119992 Москва, Россия; e-mail: irina_shevtsova@mail.ru

1) Работа выполнена при поддержке Российского фонда фундаментальных исследований, проекты 05-01-0039906, 05-07-90103. 